\title{
RADIATION PRESSURE IN STELLAR ATMOSPHERES WITH APPLICATION TO SOLAR SPICULES
}

\author{
R. GRANT ATHAY \\ High Altitude Observatory, National Center for Atmospheric Research*, Boulder, Colo., U.S.A.
}

\begin{abstract}
Radiation pressure due to absorption in spectral lines can play an important role in the equilibrium conditions in the outer portions of the stellar atmosphere. In particular, it is suggested that spicules ejected from the upper solar chromosphere as well as other fine structural features of the chromosphere may very likely be a result of radiation pressure from $L y-\alpha$. Numerical computation of the radial radiation

pressure due to Ly- $\alpha$ in the chromospheric model of Vernazza et al. (1973) gives a maximum outward pressure gradient due to radiation that is 0.06 of the gravitational pressure gradient. The model chromosphere of Vernazza $e t$ al. is a spherically symmetric model that reproduces the average Sun $\mathrm{Ly}-\alpha$ intensity and profile. Supergranule borders (network), where spicules arise, are known to have a Ly- $\alpha$ brightness some ten times the average Sun brightness. It is suggested, therefore, that in the network areas the pressure gradient due to radiation is comparable to (and oppositely directed to) that due to gravitation and that in local areas of unusual brightness the net pressure gradient is outwards. An estimate of the expected outwards velocity of spicule material driven by radiation pressure was made by equating the kinetic energy flux $\left(\frac{1}{2} \varrho V^{3}\right)$ to the Ly- $\alpha$ energy flux. This gives values of $v$ that are comparable to observed spicule velocities. Also, the maximum outwards radiation pressure occurs in the upper chromosphere where the material density in the model of Vernazza et al. is close to the value $4 \times 10^{-14} \mathrm{~g} \mathrm{~cm}^{-3}$, which is about a factor of two less than the currently accepted value for spicules. Increased density in the network areas could easily remove this minor difference.

A downward radiation pressure gradient due to Ly- $\alpha$ occurs in the model of Vernazza et al. at heights below $2250 \mathrm{~km}$. The maximum downward radiation pressure gradient in the spherically symmetric model is 0.006 of the gravitational pressure gradient. Again, this value may be increased in the network areas by well over a factor of ten and, together with the lateral gradient in radiation pressure in the network, may help give rise to much of the chromospheric fine structure associated with the network.
\end{abstract}

The author is indebted to J. E. Vernazza, E. H. Avrett and R. Loeser for supplying details of their Ly- $\alpha$ computations that were used for computing the radiation pressure.

\section{Reference}

Vernazza, J. E., Avrett, E. H., and Loeser, R.: 1973, Astrophys. J. 184, 605.

* The National Center for Atmospheric Research is sponsored by the National Science Foundation. 


\title{
DISCUSSION
}

There is some disagreement as to the significance of the $L y-\alpha$ radiation pressure as an important factor in the dynamics of spicules (Beckers, Pecker). The following items were discussed in particular:

\section{(i) To What Extent does this Model Reproduce the Observed Spicule Properties?}

\begin{abstract}
Athay's model refers to a one-dimensional, spherically symmetric atmosphere. To what extent can its properties be used to preduct spicule properties? It seems that one of the first requirements is the presence of small bright $\mathrm{Ly}-\alpha$ points with $100 \times$ the average brightness (Delache, Beckers, Giovanelli). These bright points may be caused by conduction downward from the corona along a magnetic flux tube (Athay). Given these small bright points, why does radiation pressure cause the sudden beginning of a spicule and why does it cause its very elongated cylindrical shape (Sturrock)? The properties can really only be predicted after the full dynamic model has been worked out (Athay). The absence of this full dynamic calculation led to other unanswered questions like: (a) how long does it take to build up a spicule (Delache), (b) what is the influehce of drag forces (Pecker), and (c) how much energy is needed to overcome the gravitational potential (Schmidt)? (See Section (iv).)
\end{abstract}

\section{(ii) Coupling of Neutral Hydrogen Atoms to the Gas}

The radiation pressure of $L y-\alpha$ works in the first instance only on the neutral hydrogen atoms. Are there enough collisions to transfer their momentum to the plasma as a whole (Brandt)? The answer seems affirmative (Athay).

\section{(iii) Radiation Pressure vs Electron Pressure}

Giovanelli pointed out that radiation pressure and electron pressure are related concepts. Photon generation results from electron collisions, so that for a given total energy an increase in radiation pressure must be accompanied by a reduction in electron pressure. Their combined effects should be more or less equivalent except for numerical differences between photon and electron properties such as the mean free path. Radiation pressure at small Ly- $\alpha$ optical depths ( $\tau \leqslant 3$ according to Athay) is very anisotropic in contrast to the electron pressure (Gabriel). Therefore it can be used as an acceleration mechanism.

\section{(iv) The Effect of Gravity on the Model}

Schmidt pointed out that you have to include the work done against gravity in lifting the spicule material in order to compute a realistic expected velocity. This was already pointed out in a review paper by Pikel'ner. Most of the energy flux is actually used this way rather than as a kinetic energy flux. The resultant spicule velocity becomes consequently much smaller. Athay agreed with the importance of this effect, which he had inadvertently overlooked, and noted that it will change the conclusions substantially. 